Josie Rodrigues Ferrão Quintella

\title{
Simulação da Injeção Alternada Água-Emulsão como Processo de Recuperação Avançada de Petróleo
}

Dissertação apresentada como requisito parcial para obtenção do grau de Mestre pelo Programa de Pós-graduação em Engenharia Mecânica do Departamento de Engenharia Mecânica da PUC-Rio

Orientador : Prof. Márcio da Silveira Carvalho Co-Orientador: Dra. Ranena Verónica Ponce Flores 

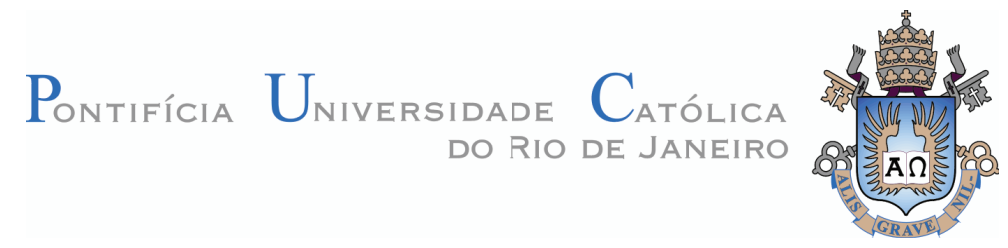

Josie Rodrigues Ferrão Quintella

\title{
Simulação da Injeção Alternada Água-Emulsão como Processo de Recuperação Avançada de \\ Petróleo
}

Dissertação apresentada como requisito parcial para obtenção do grau de Mestre pelo Programa de Pós-graduação em Engenharia Mecânica do Departamento de Engenharia Mecânica do Centro Técnico Científico da PUC-Rio. Aprovada pela Comissão examinadora abaixo assinada.

\author{
Prof. Prof. Márcio da Silveira Carvalho \\ Orientador \\ Departamento de Engenharia Mecânica - PUC-Rio \\ Prof. Dra. Ranena Verónica Ponce Flores \\ Co-Orientador \\ Coordenação de Mecânica Computacional — LNCC
}

Prof. Prof. Eurípedes Vargas

Departamento de Engenharia Civil - PUC-Rio

Prof. Dr. Antônio Luiz Serra de Souza

PETROBRAS

Prof. Prof ${ }^{a}$. Mônica Feijó Naccache

Departamento de Engenharia Mecânica - PUC-Rio

Prof. Prof. José Eugenio Leal

Coordenador Setorial do Centro Técnico Científico - PUC-Rio 
Todos os direitos reservados. É proibida a reprodução total ou parcial do trabalho sem autorização da universidade, do autor e do orientador.

\section{Josie Rodrigues Ferrão Quintella}

Graduou-se em Engenharia Mecânica pela Universidada Federal do Rio de Janeiro em 2002. Atualmente atua na área de regulação de petróleo, na Agência Nacional do Petróleo, Gás Natural e Biocombustíveis.

Ficha Catalográfica

Quintella, Josie Rodrigues Ferrão

Simulação da Injeção Alternada Água-Emulsão como Processo de Recuperação Avançada de Petróleo / Josie Rodrigues Ferrão Quintella; orientador: Prof. Márcio da Silveira Carvalho; co-orientador: Dra. Ranena Verónica Ponce Flores. - Rio de Janeiro : PUC-Rio, Departamento de Engenharia Mecânica, 2012.

v., 65 f: il. ; $29,7 \mathrm{~cm}$

1. Dissertação (mestrado) - Pontifícia Universidade Católica do Rio de Janeiro, Departamento de Engenharia Mecânica.

Inclui referências bibliográficas.

1. Engenharia Mecânica - Tese. 2. Emulsão; Recuperação de Petróleo; Controle de Mobilidade; Simulação de Reservatórios.. I. Carvalho, Márcio da Silveira. II. Ponce Flores, Ranena Verónica. III. Pontifícia Universidade Católica do Rio de Janeiro. Departamento de Engenharia Mecânica. IV. Título. 
À minha família. 


\section{Agradecimentos}

Agradeço à minha família, em especial a minha mãe, meu pai e minha irmã, pela formação, carinho e por ser meu suporte de todos os momentos. Ao ao meu marido, Leonardo, pelo companheirismo em todas as etapas deste trabalho. Ao meu orientador, Professor Márcio da Silveira Carvalho, pela confiança, apoio e direcionamento durante a realização deste trabalho. À minha co-orientadora, Dra. Ranena Verónica Ponce Flores, pela paciência, parceria e orientação constante e fundamental para a conclusão desta dissertação. Aos profissionais do GReo e do Laboratório de Microhidrodinâmica e Escoamento em Meios Porosos pela amizade e ajuda que sempre me dedicaram. Ao Departamento de Engenharia Mecânica da Pontifícia Universidade Católica do Rio de Janeiro pelos ensinamentos e oportunidade de realizar este trabalho, valorizando minha formação profissional. À Banca Examinadora, pelas observações contribuídas ao presente trabalho. 


\section{Resumo}

Quintella, Josie Rodrigues Ferrão; Carvalho, Márcio da Silveira; Ponce Flores, Ranena Verónica. Simulação da Injeção Alternada ÁguaEmulsão como Processo de Recuperação Avançada de Petróleo. Rio de Janeiro, 2012. 65p. Dissertação de Mestrado - Departamento de Engenharia Mecânica, Pontifícia Universidade Católica do Rio de Janeiro.

A injeção de emulsão óleo-água vem sendo estudada como método de recuperação avançada, levando a um incremento no volume de óleo recuperado através de um controle de mobilidade do fluido injetado e aumento da eficiência de deslocamento do óleo. A aplicação deste método requer o entendimento do escoamento tanto na escala de poros como na escala de reservatórios. Neste trabalho, o efeito da emulsão no escoamento bifásico é modelado através de curvas de permeabilidades relativas que variam com a concentração de gotas da fase dispersa da emulsão. Estas curvas descrevem dois fenômenos físicos observados nestes escoamentos, o controle de mobilidade, representado pela diminuição da permeabilidade relativa da fase aquosa devido à presença de gotas, e a melhora na eficiência de deslocamento, representada pela diminuição da saturação residual da fase oleosa. O modelo proposto foi implementado em um simulador comercial (STARS-CMG) em uma geometria bi-dimensional com dois poços verticais (injetor e produtor). A produção de óleo durante o processo de injeção alternada de água/emulsão/água é estudado em diversas condições do escoamento com o objetivo de analisar separadamente o mecanismo de controle de mobilidade e da melhoria do deslocamento no processo, determinando as condições ótimas de operação. Os resultados obtidos mostram que a injeção de emulsão óleo-água pode representar uma importante alternativa na produção de óleo como processo de recuperação avançada.

\section{Palavras-chave}

Emulsão; Recuperação de Petróleo; Controle de Mobilidade; Simulação de Reservatórios. 


\section{Abstract}

Quintella, Josie Rodrigues Ferrão; Carvalho, Márcio da Silveira (Advisor); Ponce Flores, Ranena Verónica (Co-Advisor). Numerical Simulation of Alternating Water-Emulsion Injection as an Enhanced Oil Recovery Process. Rio de Janeiro, 2012. 65p. MSc. Dissertation Departamento de Engenharia Mecânica, Pontifícia Universidade Católica do Rio de Janeiro.

Emulsion injection has been studied as an enhanced oil recovery method, leading to an increase on the recovered oil volume by mobility control of injected fluid and increase of oil displacement efficiency. The use of this method requires understanding the flow both in pore and reservoir scales. The effect of emulsion on the two phase flow is modeled by using relative permeability curves that vary with the concentration of the dispersed phase of the emulsion. Those curves describe two physical phenomena observed on these flows: control of mobility, represented by a lower relative permeability of the aqueous phase due to the presence of droplets and improved displacement efficiency, represented by the decrease in the residual oil saturation. The proposed model was implemented in a commercial simulator (STARS-CMG) in a two-dimensional geometry with two vertical wells (injector and producer). The oil production during alternate injection of water/emulsion/water process is studied with several flows condition with the purpose to analyze separately the effect of mobility control and improvement of the oil displacement in the process and finding the optimum operating conditions. The results obtained show that the oil-water emulsion injection can represent an important alternative in oil production as an enhanced oil recovery method.

\section{Keywords}

Emulsion; Oil Recovery; Mobility Control; Reservoir Simulation. 


\section{Sumário}

$\begin{array}{lr}\text { Lista de Símbolos } & 14\end{array}$

1 Introdução $\quad 16$

$\begin{array}{ll}1.1 \text { Motivação } & 16\end{array}$

$\begin{array}{lll}1.2 & \text { Objetivo } & 18\end{array}$

$\begin{array}{lll}1.3 & \text { Roteiro } & 18\end{array}$

2 Processos de Recuperação de Óleo 20

2.1 Introdução 20

2.2 Recuperação Primária $\quad 21$

2.3 Recuperação Secundária $\quad 21$

2.4 Eficiência de um Projeto de Injeção de Fluidos 21

2.5 Recuperação Avançada 24

2.6 Injeção de Emulsão 25

3 Escoamento em Meios Porosos $\quad 31$

$\begin{array}{lll}3.1 & \text { Fluidos no meio poroso } & 31\end{array}$

$\begin{array}{ll}3.2 & \text { Lei de Darcy } \\ 3.3 & 31\end{array}$

3.3 Equação da Continuidade 32

3.4 Equação de Estado 33

3.5 Equação da Difusividade Hidráulica 36

3.6 Escoamento Bifásico em Meios Porosos 37

3.7 Modelo de Escoamento de Emulsão 40

4 Abordagem Numérica $\quad 43$

4.1 Apresentação do Simulador Numérico 43

4.2 Modelagem do Reservatório 44

4.2.1 Malha do Reservatório 44

4.2.2 Propriedades da Rocha 45

4.2.3 Propriedades dos Fluidos 45

4.2.4 Condições de Operação 46

4.3 Descrição dos Casos Simulados 46

4.3.1 Caso 1: Injeção Contínua de Água 46

4.3.2 Caso 2: "Caso Base" 47

4.3.3 Caso 3: Efeito do volume poroso de emulsão injetada 47

4.3.4 Caso 4: Efeito do tempo de início de injeção de emulsão 47

4.3.5 Caso 5: Efeito dos parâmetros das curvas de permeabilidade relativa da emulsão injetada $\quad 47$

4.3.6 Caso 6: Efeito da Heterogeneidade do Meio Poroso 48

5 Resultados e Discussões $\quad 51$

5.1 Comparação entre os Mecanismos $\quad 51$

5.2 Efeito do volume poroso de emulsão injetada 54

5.3 Efeito do tempo de início de injeção de emulsão 55 
5.4 Efeito dos parâmetros das curvas de permeabilidade relativa da emulsão injetada

5.4.1 Mecanismo de Controle da Mobilidade 57

5.4.2 Mecanismo de Eficiência de Deslocamento 59

5.4.3 Efeito da Heterogeneidade do Meio Poroso 60

6 Considerações Finais $\quad 62$

$\begin{array}{ll}\text { Referências Bibliográficas } & 64\end{array}$ 


\section{Lista de figuras}

1.1 Consumo por região e R/P de óleo (Fonte: Anuário Estatístico $\mathrm{BP} / 2011)$.

1.2 Consumo por região e R/P de gás (Fonte: Anuário Estatístico $\mathrm{BP} / 2011)$.

1.3 Histórico e projeção de preços do petróleo (Fonte: EIA - International Energy Outlook 2011).

2.1 Matriz rochosa e espaço poroso [1].

2.2 Canalização durante o processo de injeção de água em um reservatório [2].

2.3 Evolução da área invadida sujeito à injeção de água [3].

2.4 Seção vertical de um reservatório estratificado sujeito à injeção de água [4].

2.5 Comportamento do fator de redução da mobilidade $(f)$ [5].

2.6 Variação do fator de redução de mobilidade $(f)$ em função do número capilar [6].

2.7 Evolução do volume de óleo recuperado e pressão de injeção obtidos adimensionalmente com número capilar de $2 \times 10^{-5}$ [7].

2.8 Evolução do volume de óleo recuperado e pressão de injeção obtidos adimensionalmente com número capilar $2 \times 10^{-4}$ [7].

2.9 Evolução do volume de óleo recuperado e pressão de injeção obtidos durante a injeçào alternada de água e emulsão em amostras de rochas paralelas de diferentes permeabilidades. $O$ sombreado verde indica o período de injeção de emulsão [8].

3.1 Elemento do meio poroso macroscópico para o desenvolvimento da equação de conservação de massa.

3.3 Distribuição de fluidos em um sistema molhado preferencialmente pela água.

3.4 Permeabilidades Relativas Sistema Água-Óleo.

3.5 Permeabilidades Relativas Água-Óleo $\left(k_{r w}, k_{r_{o_{w}}}\right)$ e Emulsão-Óleo $\left(k_{r e}, k_{r o_{e}}\right)$ - Mecanismo Controle da Mobilidade.

3.6 Permeabilidades Relativas Água-Óleo $\left(k_{r w}, k_{r_{w}}\right)$ e Emulsão-Óleo $\left(k_{r e}, k_{r o_{e}}\right)$ - Mecanismo Eficiência de Deslocamento.

4.1 Malha do modelo do reservatório.

4.2 Mapa de permeabilidade absoluta.

4.3 Curvas de kr utilizadas na análise paramétrica do fator de bloqueio - Emulsão com Mecanismo de Controle de Mobilidade.

4.4 Curvas de kr utilizadas na análise paramétrica da Sor - Emulsão com Mecanismo de Deslocamento.

4.5 Quadro sinótico dos casos estudados. 
5.1 Simulação da injeção alternada A/E/A simulados para o Caso Base - A) Mecanismo de controle da mobilidade; B) Mecanismo de eficiência de deslocamento.

5.2 Mapas de Saturação de Água (Sw) durante o processo de injeção alternada A/E/A simulados para o "Caso Base".

5.3 Efeito do volume poroso de emulsão injetada no fator de recuperação de óleo durante o processo de injeção alternada $A / E / A$ - A) Mecanismo de controle da mobilidade; B) Mecanismo de eficiência de deslocamento.

5.4 Mapas de Saturação de Água (Sw) durante o processo de injeção alternada A/E/A simulados para os casos 3 - Mecanismo de Controle de Mobilidade.

5.5 Mapas de Saturação de Água (Sw) durante o processo de injeção alternada A/E/A simulados para os casos 3 - Mecanismo de Eficiência de Deslocamento.

5.6 Efeito do tempo de início da injeção de emulsão no fator de recuperação de óleo durante o processo de injeção alternada $A / E / A$ - A) Mecanismo de controle da mobilidade; B) Mecanismo de eficiência de deslocamento.

5.7 Efeito do fator de bloqueio da emulsão injetada no fator de recuperação de óleo durante o processo de injeção alternada A/E/A. 60

5.8 Efeito da mudança da Sor no fator de recuperação de óleo durante o processo de injeção alternada $A / E / A$.

5.9 Efeito da heterogeneidade do reservatório no fator de recuperação durante o processo de injeção alternada A/E/A - A) Mecanismo de controle da mobilidade; B) Mecanismo de eficiência de deslocamento. 61 


\section{Lista de tabelas}

4.1 Variação da Permeabilidade Absoluta. 


\section{Lista de Símbolos}

\begin{tabular}{|c|c|}
\hline$\lambda_{f}$ & Mobilidade do fluído \\
\hline$\mu_{f}$ & Viscosidade do fluído \\
\hline$\rho_{f}$ & Massa específica do fluído \\
\hline$\phi$ & Porosidade da rocha \\
\hline$A$ & Área da seção transversal \\
\hline$A / E / A$ & Injeção alternada água-emulsão-água \\
\hline$A_{i n v}$ & Área invadida pelo fluido injetado \\
\hline$A_{t}$ & Área total \\
\hline$B$ & Fator volume de formação \\
\hline$B_{g}$ & Fator volume de formação do gás \\
\hline$B_{o}$ & Fator volume de formação do óleo \\
\hline$B_{w}$ & Fator volume de formação da água \\
\hline$c$ & Compressibilidade isotérmica do fluido \\
\hline$c_{r}$ & Compressibilidade da rocha \\
\hline$c_{t}$ & Compressibilidade total \\
\hline$E_{A}$ & Eficiência de varrido horizontal \\
\hline$E_{D}$ & Eficiência de deslocamento \\
\hline$E_{R}$ & Eficiência de recuperação \\
\hline$E_{V V}$ & Eficiência de varrido vertical \\
\hline f & Fator de redução de mobilidade \\
\hline$f_{b}$ & Fator de redução de mobilidade \\
\hline$F R$ & Fator de recuperação \\
\hline$g$ & Aceleração da gravidade \\
\hline$k$ & Permeabilidade absoluta \\
\hline$k_{f}$ & Permeabilidade efetiva \\
\hline$k_{r}$ & Permeabilidade relativa \\
\hline$k_{r e}$ & Permeabilidade relativa à emulsão \\
\hline$k_{r e\left(S_{o r}\right)}$ & Permeabilidade relativa à emulsão na saturação de óleo residual \\
\hline$k_{r i}$ & Permeabilidade relativa correspondente a fase "i", \\
\hline$k_{r o_{e}}$ & Permeabilidade relativa ao óleo para o sistema emulsão-óleo \\
\hline$k_{r o_{w}}$ & Permeabilidade relativa ao óleo para o sistema água-óleo \\
\hline$k_{\text {rowi }}$ & Permeabilidade relativa ao óleo máxima \\
\hline$k_{r w}$ & Permeabilidade relativa à água \\
\hline$k_{r w(S o r)}$ & Permeabilidade relativa à água na saturação de óleo residual \\
\hline$k_{\text {rwor }}$ & Permeabilidade relativa à água máxima \\
\hline$M$ & Razão de mobilidades \\
\hline$m_{i}$ & Massa do componente i \\
\hline
\end{tabular}




\begin{tabular}{|c|c|}
\hline$n_{o}$ & Expoente de Corey para o óleo \\
\hline$n_{w}$ & Expoente de Corey para a água \\
\hline$p$ & Pressão \\
\hline$p_{\operatorname{cog}}$ & Pressão capilar contato óleo-gás \\
\hline$p_{\text {cow }}$ & Pressão capilar contato óleo-água \\
\hline$q$ & Vazão volumétrica \\
\hline$R_{s}$ & Razão de solubilidade \\
\hline$S_{f}$ & Saturação do fluido \\
\hline$S_{o}$ & Saturação do óleo \\
\hline$S_{o i}$ & Saturação do óleo no início da injeção \\
\hline$S_{o r}$ & Saturação do óleo residual \\
\hline $\bar{S}_{o}$ & Saturação média de óleo ao final do processo \\
\hline$S_{w}$ & Saturação da água \\
\hline$S_{w i}$ & Saturação da água conata ou irredutível \\
\hline$S_{w}$ & Saturação da água \\
\hline$v$ & Velocidade \\
\hline$V_{d g}$ & Volume de gás dissolvido no óleo \\
\hline$V_{f}$ & Volume do fluido \\
\hline$V_{o}$ & Volume de óleo \\
\hline$V_{p}$ & Volume poroso \\
\hline$V_{t}$ & Volume total \\
\hline$V_{w}$ & Volume de água \\
\hline$W C U T$ & Corte de água \\
\hline$z$ & Distância vertical \\
\hline
\end{tabular}


Se o conhecimento pode criar problemas, não é através da ignorância que podemos solucionálo.

Isaac Asimov, 